\title{
An automated home-cage-based 5-choice serial reaction time task for rapid assessment of attention and impulsivity in rats
}

\author{
B. Bruinsma ${ }^{1} \cdot$ H. Terra ${ }^{1} \cdot$ S. F. de Kloet ${ }^{1} \cdot$ A. Luchicchi $^{2} \cdot$ A. J. Timmerman ${ }^{1} \cdot$ E. Remmelink $^{3} \cdot$ M. Loos $^{3} \cdot$ Tommy Pattij $^{2} \cdot$ \\ Huibert D. Mansvelder ${ }^{1}$
}

Received: 23 May 2018 / Accepted: 4 February 2019 / Published online: 2 March 2019

(C) The Author(s) 2019

\begin{abstract}
Rationale The 5-choice serial reaction time task (5-CSRTT) is a widely used operant task for measuring attention and motor impulsivity in rodents. Training animals in this task requires an extensive period of daily operant sessions. Recently, a self-paced, automated version of this task has been developed for mice, which substantially reduces training time. Whether a similar approach is effective for rats is currently unknown.

Objective Here, we tested whether attention and impulsivity can be assessed in rats with a self-paced version of the 5-CSRTT. Methods Operant boxes were connected to home-cages with tunnels. Two groups of rats self-paced their training by means of an automated script. The first group of animals was allowed unlimited access (UA) to start trials in the task; for the second group, trial availability was restricted to the first $2.5 \mathrm{~h}$ of the dark cycle (TR). Task parameter manipulations, such as variable inter-trial intervals and stimulus durations as well as pharmacological challenges with scopolamine, were tested to validate the task.

Results Self-paced training took less than 1 week. Animals in the UA group showed higher levels of omissions compared with the TR group. In both protocols, variable inter-trial intervals increased impulsivity, and variable stimulus durations decreased attentional performance. Scopolamine affected cognitive performance in the TR group only.

Conclusions Home-cage-based training of the 5-CSRTT in rats, especially the TR protocol, presents a valid and fast alternative for measuring attention and impulsivity.
\end{abstract}

Keywords 5 -CSRTT $\cdot$ Rats $\cdot$ Home-cage $\cdot$ Attention $\cdot$ Impulsivity $\cdot$ Scopolamine $\cdot$ Animal model

\section{Introduction}

Animal models of executive functioning are pivotal to understanding the neurobiology of psychiatric illness. Executive function domains, such as attention and impulse control, are

B. Bruinsma, H. Terra and S. F. de Kloet contributed equally.

T. Pattij and H.D. Mansvelder have shared senior authorship.

Tommy Pattij

t.pattij@vumc.nl

Huibert D. Mansvelder

h.d.mansvelder@vu.nl

1 Department of Integrative Neurophysiology, Center for Neurogenomics and Cognitive Research (CNCR), Amsterdam Neuroscience, VU University, Amsterdam, The Netherlands

2 Department of Anatomy and Neurosciences, Amsterdam Neuroscience, VU University Medical Center, Amsterdam, The Netherlands

3 Sylics (Synaptologics B.V.), Amsterdam, The Netherlands affected in several psychiatric disorders, including schizophrenia and attention-deficit hyperactivity disorder (ADHD) (Castellanos and Tannock 2002; Luck and Gold 2008). The 5-choice serial reaction time task (5-CSRTT) is a widely utilized behavioral paradigm for rodents to test visual sustained attention and motor impulsivity (Robbins 2002; Blondeau and Dellu-Hagedorn 2007). In this task, animals are trained to scan a horizontal array of 5 apertures for the onset of a visual stimulus and withhold responding until its appearance. After a stimulus presentation in one of the pseudo-randomly chosen apertures, the animal must make a response in the form of a nose poke within a limited time window. From typically 60 to 100 repetitions of these trials, attentional performance is deduced from the ratio of the number of correct and incorrect responses. Levels of motor impulsivity can be assessed from the number of premature responses before the onset of the visual cue. Importantly, possible non-specific effects of pharmacological or neuronal circuit interventions can be controlled for by assessing motor effects via different response latencies (Robbins 2002; Bari et al. 2008). 
Before animals can perform this task reliably with a stimulus duration (SD) of typically 0.5 to $1.0 \mathrm{~s}$, weeks to months of operant training are required (Bari et al. 2008; Bhandari et al. 2016). Not only is this labor-intensive, the long periods of food deprivation can add to the cumulative discomfort of animals during the experiment. Besides animal discomfort, idiosyncratic handling by the experimenter has been shown to alter behavioral outcomes in rats, such as learning and memory (Bohacek and Daniel 2007). Additionally, experimenterinduced interventions can increase corticosterone concentrations (Sorge et al. 2014; Deutsch-Feldman et al. 2015), which in turn could affect executive functioning (Sänger et al. 2014).

A previous study asserted the efficacy of a self-paced variant of the 5-CSRTT (SP-5-CSRTT) in mice. In that study, home-cages of animals were connected to operant 5-CSRTT chambers (the so-called CombiCage), and mice could selfpace task progression with minimal interference by experimenters (Remmelink et al. 2017). This adaptation of the 5CSRTT led to a marked reduction in time that animals took to learn the task. Although the researchers reported slight differences in baseline performance at a SD of $1 \mathrm{~s}$ between animals trained in the SP-5-CSRTT and a conventional 5-CSRTT protocol, effects of behavioral challenges on attention and impulse control were similar. Additionally, the use of the SP-5CSRTT for drug testing was shown by a dose-dependent effect of scopolamine, an acetylcholine muscarinic receptor antagonist, on attentive behavior (Remmelink et al. 2017). Whether this approach could be applied to testing attention and impulse control in rats is unknown. Additionally, whether task availability in the home-cage setting is an important factor for learning speed and performance is still unknown.

Here, we tested a modified version of the CombiCage SP5-CSRTT, which was adjusted for rats. We measured training time and baseline performance and validated the SP-5-CSRTT by randomly varying behavioral parameters and quantifying effects on attention and impulsivity. Finally, we tested the effects of scopolamine, a muscarinic acetylcholine receptor antagonist, which has been shown to impact attention and impulsivity in rats in the conventional 5-CSRTT (Robbins 2002).

\section{Methods}

\section{Animals}

For training and testing in CombiCages, 36 male Long Evans rats (Janvier Labs, France, 8 weeks old) were initially housed in pairs with food and water available ad libitum 1 week before the start of experiments. Next, animals were housed individually in CombiCages, and behavioral procedures were initiated. Rats were housed under a 12-h light/dark cycle (lights off at 12 PM). For the conventional 5-CSRTT training, 14 male Long Evans rats (Janvier Labs, France, 8 weeks old) were housed individually. Food restriction began 1 week prior to behavioral training to achieve and maintain $85-90 \%$ of free feeding weight. Animals were trained daily for 5 days per week (Monday-Friday) as described in Luchicchi et al. (2016). One animal in the time-restricted (TR) group became sick after training and variable-ITI sessions and was excluded for the variable-SD session and scopolamine experiments. All experimental procedures were in accordance with the European and Dutch law and approved by the animal ethical care committee of the VU University and VU University Medical Center.

\section{SP-5-CSRTT task}

For construction of CombiCages, a standard makrolon homecage was connected to an operant box (Med-Associates Inc., St. Albans, VT, USA) with a custom-made polymer tube with a diameter of $10 \mathrm{~cm}$. Operant chambers were on one side equipped with five cue holes, containing LED stimulus lights and infrared beam detectors. On the opposite wall, a food magazine, a red magazine light, and a yellow houselight were placed (Fig. 1).

Rats were placed in CombiCages 2 days before the experiment started, and food was available ad libitum. After the start of the task, animals earned their food in the form of pellets in the task (Dustless Precision Pellets, grain-based, F0165, $45 \mathrm{mg}$, Bio-Serve, USA). Animals were weighed each day before onset of the dark cycle. Animals were not food restricted prior to the start of the training. If rats did not earn enough pellets to gain weight according to an $85-90 \%$ food restriction regime, additional chow was given. In the present study, no additional chow was necessary during training, animals in the TR-group were fed extra chow after training to keep stable grow and performance during pharmacological testing.

For training in the SP-5-CSRTT, the same training stages were applied as in conventional 5-CSRTT training (Remmelink et al. 2017). First, animals learned to associate pellet delivery with reward during magazine training, and during 50 trials, a pellet was delivered after a variable inter-trial interval (ITI) of 4, 8, 16, or $32 \mathrm{~s}$. Reward availability in the task was signaled by the magazine light, and collection of pellets triggered the next trial start. In the subsequent training stage, all five stimulus lights were lit until a nose-poke response was made in one of them to earn a reward. After 50 trials, animals moved on to the next stage. Here, a nose-poke response in the food magazine started an ITI period of $5 \mathrm{~s}$ followed by presentation of randomly selected single stimulus light. A nose poke into the lit cue hole was rewarded with a pellet; incorrect nose pokes were not punished.

In the next stage, rats started trials with a nose poke in the food magazine, starting an ITI of $5 \mathrm{~s}$. Subsequently, one of the 5 cue holes was lit for a certain SD. Initially, SDs were $16 \mathrm{~s}$ 
Fig. 1 Schematic overview of the rat CombiCage. A standard macrolon home-cage is connected to a Med-Associates operant box by a polymer connection tube (diameter $10 \mathrm{~cm}$ ). The operant box is equipped with a food magazine connected to a pellet dispenser. On the opposite wall five equally spaced cue holes are positioned with yellow LEDs. Each cue hole is equipped with an infrared response detector to measure nose-poke responses
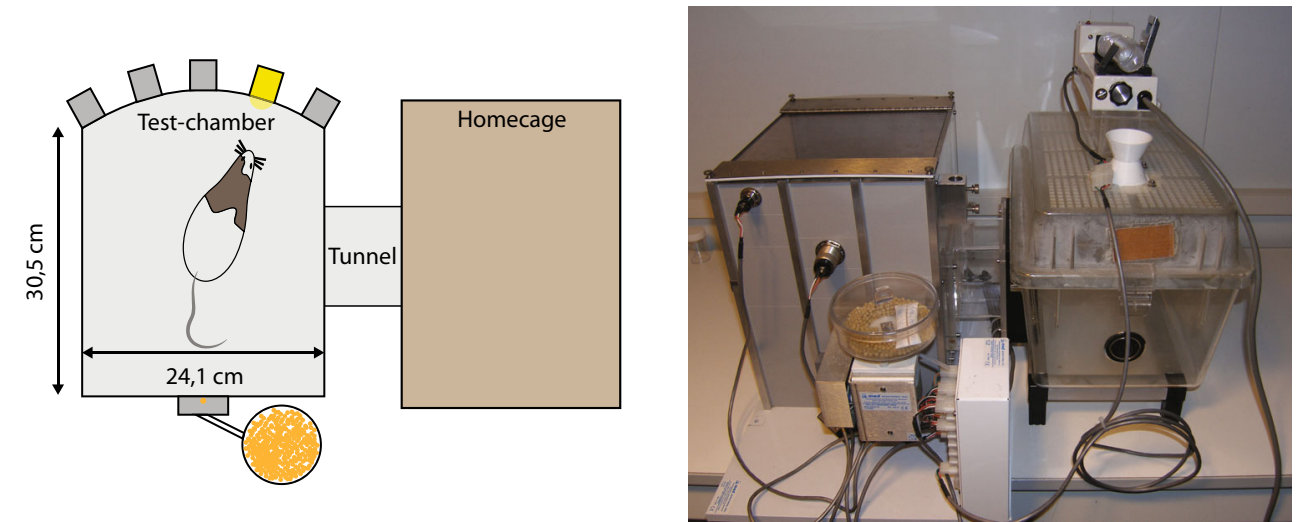

and were titrated down in five steps to $1 \mathrm{~s}$ for the final stage. Rats had to make a response in the lit stimulus hole during stimulus presentation or within a 2 -s limited hold period after stimulus presentation. A lack of response was considered an omission and resulted in a time-out period of $5 \mathrm{~s}$. Incorrect and premature responses, during the ITI, also resulted in a time-out period of $5 \mathrm{~s}$. Additionally, these errors were signaled with the houselight that was switched on for the duration of the timeout period. Correct responses were rewarded with a food pellet. After reward collection in the food magazine, rats could start the next trial $5 \mathrm{~s}$ later with a subsequent nose poke in the food magazine. We refer to the period of reward collection before start of the next trial as the "eat-interval."

For the SP-5-CSRTT protocols, the performance criterion to reach a following stage with shorter $\mathrm{SD}$ was a minimum of 50 started trials with accuracy levels (ratio of correct and incorrect responses, see below) $>80 \%$ and either omissions $<20 \%$ or number of correct trials $>200$ in the current stage. These parameters were calculated online during task performance using a sliding window of 20 trials on which accuracy levels and percentage omissions were calculated. This approach was based on recent work in mice (Remmelink et al. 2017). If the animal passed the performance criterion in this block of 20 trials analyzed by the sliding window, the program automatically moved to the next stage (Remmelink et al. 2017).

Two different groups were trained in CombiCages with different trial availabilities. In the unlimited access (UA) protocol, animals could initiate trials $24 \mathrm{~h}$ per day, whereas in the TR protocol, rats could only start trials during the first $2.5 \mathrm{~h}$ of the dark cycle. To examine effects of manipulation of task parameters, both groups were subjected to a session with variable ITIs $(5,7.5$, or $12.5 \mathrm{~s})$ or variable SDs $(0.2,0.5$, or $1 \mathrm{~s})$. These sessions comprised of a block of 500 trials for the UA group and a 2.5 -h session for the TR group.

In the conventional 5-CSRTT group, rats were trained in the same training stages as described for the home-cage protocols. The criterion to move on to the next stage was set at accuracy $>80 \%$ and omissions $<20 \%$. Performance was calculated after each half-hour session.

\section{Drug administration}

Scopolamine hydrochloride (Sigma-Aldrich, St. Louis, MO, USA) was dissolved in $0.9 \%$ saline and injected intraperitoneally (i.p.) $20 \mathrm{~min}$ prior to the start of the dark phase. Scopolamine was freshly prepared on each test day, and doses were administered using a Latin square design on Monday, Wednesday, and Friday. Animals continued with training on Tuesday, Thursday, Saturday, and Sunday.

\section{Data analysis and statistics}

All data were acquired with MED-PC software (MedAssociates, USA). Data analyses and statistics were done with custom-written scripts in MATLAB (Mathworks, USA). Accuracy was calculated a: (\#correct) / ( \#correct + \#incorrect) * 100). Omissions and premature responses were expressed as percentage of the total number of trials. Correct-response latency was expressed as the time in seconds between stimulus onset and a correct response. Magazine latency was expressed as the time in seconds between the correct response and pellet collection. Trials with a magazine latency $>10$ s were excluded from further analysis. Normality of the data was tested with the Shapiro-Wilk test.

For comparison of training time and the number of required trials per training stage in the different groups, a Wilcoxon rank-sum test and two-way mixed repeated-measures ANOVA were used with group as between-subjects factor. Post hoc testing was performed using Wilcoxon rank-sum tests or $t$ tests with Benjamin-Hochberg false discovery rate (FDR) to adjust $p$ values for multiple comparisons (Benjamini and Hochberg 1995).

To compare baseline performance between groups, a block of 500 trials at SD1 for the UA group after passing SD1 criterion was compared with a 2.5-h session of SD1 trials for the TR group. Additionally, we compared baseline performance of the first 100 trials of the dark cycle for the TR and UA protocol with the CT baseline session. For both analyses, $t$ tests or Wilcoxon rank-sum tests were performed. 
Behavioral challenges with variable ITI or SD were only performed in the SP-5-CSRTT protocols and were analyzed using two-way mixed repeated-measures ANOVAs. To test differences in the number of started trials, accuracy, premature responses, and omissions between the dark period and light period for the UA group, $t$ tests on grand means were performed.

The effect of scopolamine was tested in 2.5-h variable-ITI sessions (TR group), or data from the first $2.5 \mathrm{~h}$ in the dark cycle was analyzed (UA group). 2.5 -h sessions were split in 30-min blocks for analyses. For the different behavioral parameters, two-way mixed repeated-measures ANOVAs were employed with dose and time as within-subject factors. Post hoc testing was performed with FDR-controlled $t$ tests or Wilcoxon rank-sum tests.

In all cases, the significance level was set at $p<0.05$.

\section{Results}

\section{Training time is less than 1 week in SP-5-CSRTT}

To test whether attention and impulsivity in rats can be assessed using an automated, self-paced task, as previously described for mice (Remmelink et al. 2017), we trained two groups of rats in an automated, modified home-cage version of the 5-CSRTT. Briefly, the home-cage of the animals was connected to an operant cage with a tunnel creating a CombiCage (Fig. 1). To test whether limited trial availability would increase motivation and affect learning speed and performance of animals, two protocols were tested that differed solely in trial availability. In the first protocol, the UA group could start trials throughout light and dark cycles for $24 \mathrm{~h}$, whereas in the second protocol, the TR group could only start trials during the first $2.5 \mathrm{~h}$ of the dark cycle. Additionally, we have included data from a group of rats conventionally trained in the 5-CSRTT, by means of daily 30-min training sessions (Luchicchi et al. 2016). This data was included to show training and baseline performance of animals that were trained in a conventional 5-CSRTT in our lab.

Animals in both the UA and TR group were trained to SD 1 criterion in less than 7 days (Table 1, Fig. 2a). In particular, rats in the UA group finished training in less than 3.5 days and were quicker than the TR group (Fig. 2a; Wilcoxon rank-sum test, $p<0.01$ ). However, the total number of trials that was required to finish $\mathrm{SD} 1$ to stable baseline performance criterion was less for the TR group (Fig. $2 \mathrm{~b} ; t$ test, $p<0.01$ ). Closer inspection of the number of trials required per stage of the task did reveal differences in learning between the groups (Fig. 2c; group $\times$ stage: $F[5,110]=4.26, p<0.01)$. Specifically, learning of the final stage, SD1, required less trials for the TR group compared with the UA protocol (Fig. 2c; FDR-corrected Wilcoxon rank-sum test, $p<0.01$ ).
Stable baseline performance in the SP-5-CSRTT

Similar to mice (Remmelink et al. 2017), in rats, the UA group also started significantly more trials during the dark phase of day-night cycles (91.1\% of total) than during the light phase (Fig. 3a, $p<0.001$ ). In addition, accurate responding was higher during the dark phase (Fig. $3 \mathrm{~b}, p<0.001$ ), and omissions were lower, compared with the light phase (Fig. 3c, $p=$ 0.018 ). Surprisingly, the percentage of premature responses, a measure for motor impulsivity, was below $1 \%$ of the number of trials. Levels of premature responding did not differ between the light or dark phase (Fig. 3d, $p=0.097$ ). Since animals started trials almost exclusively during the dark phase and because of differences in task performance during the light and dark phase, we will henceforth only report behavioral parameters analyzed for trials started during the dark phase.

Next, we analyzed baseline SD1 performance across behavioral parameters and compared them between protocols (Table 1). The UA group rats started more than 800 trials per day on average, whereas the TR group started close to 400 trials (Fig. 4a). Accuracy, the measure for attention, did not differ between protocols (Fig. $4 \mathrm{~b}, t$ test, $p=0.64$ ), with rats in all groups reaching levels of approximately $85 \%$ correct choice at SD1. Interestingly, the percentage of omitted trials markedly differed between protocols (Fig. 4c, $t$ test, $p<0.001)$. UA group rats showed almost three times more omissions than the TR group. Premature responding was reduced in the UA group compared with the TR protocol (Fig. $4 \mathrm{~d}, t$ test, $p<0.05$ ). Finally, whereas correct-response latencies were slightly elevated in the UA group compared with the TR protocol (Fig. 4e, $t$ test, $p=0.04$ ), magazine latencies were comparable (Fig. $4 \mathrm{f}, t$ test, $p=0.35$ ). When we compared the first 100 trials of the UA and TR session, we only found significant differences in the percentage of omissions between the protocols (Table 1, Wilcoxon rank-sum test, $p<0.0001)$.

\section{SP-5-CSRTT performance is modulated by variable ITI and SD manipulations}

Since we observed differences in baseline performance in the SP-5-CSRTT compared with the conventional 5-CSRTT, we asked whether behavioral challenges would affect performance equally in the different protocols. For this, we subjected rats from the UA and TR group to days with either a variable ITI or a variable SD. Randomly varying the ITI between $5,7.5$, and $12.5 \mathrm{~s}$ affected accuracy to the same extent in both groups (Fig. 5a, ITI: $F[2,42]=5.18, p<0.01$; group $\times$ ITI: $F[2,22]=1.97, p=0.15$ ). However, post hoc testing revealed no significant differences in accuracy between trials with different ITI durations. The percentage of omitted trials was significantly decreased for the UA group on trials with the longest ITI (Fig. 5b, ITI: $F[2,42]=6.57, p<0.01$; group $\times$ 
Table 1 Summary of training variables and performance for conventional training and both the unlimited access and timerestricted home-cage 5CSRTT groups a

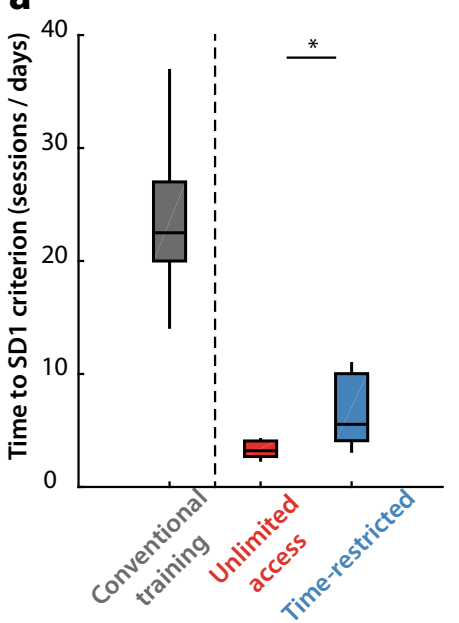

b

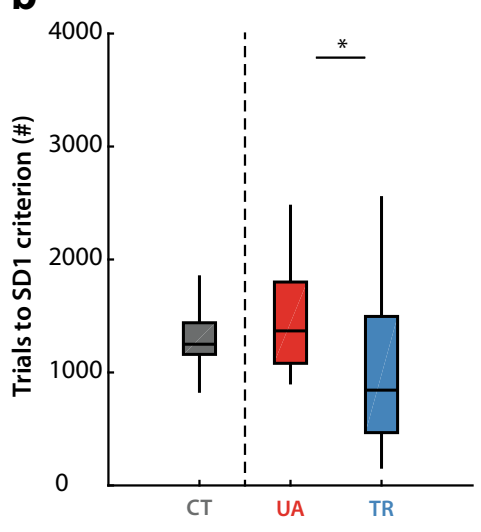

Time-restricted

Conventional training

Training

Number of rats

Days to finish SD1 criterion

Number of trials to SD1 criterion

Weight difference (\% start vs end training)

Earned pellets/day

Punishment after error

Eat-interval (s)

Performance at SD1

Started trials per session/day (\#)

Accuracy (\%)

Omissions (\%)

Premature responses (\%)

Correct-response latency (s)

Magazine latency (s)

First 100 trials SD1 session

Accuracy (\%)

Omissions (\%)

Premature responses (\%)

Correct-response latency (s)

Magazine latency (s)
Unlimited access

12

$3.29 \pm 0.76$

$1499 \pm 522$

$+2 \pm 2.8$

$282 \pm 41$

$\mathrm{TO}+\mathrm{HL}$ on

5

$832 \pm 182$

$83.11 \pm 8.12$

$49.52 \pm 8.14$

$0.12 \pm 0.08$

$1.66 \pm 0.29$

$2.21 \pm 0.47$

$80.33 \pm 11.69$

$51 \pm 10.28$

$0.33 \pm 0.49$

$1.67 \pm 0.27$

$2.09 \pm 0.42$
12

$6.55 \pm 2.99$

14

$3.21 \pm 6.13$

$347 \pm 409$

$1023 \pm 71$

NA

$268 \pm 29$

$60.71 \pm 7.97$

$\mathrm{TO}+\mathrm{HL}$ on

$\mathrm{TO}+\mathrm{HL}$ off

5

0

$00 \pm 0$

$390 \pm 60$

$84.61 \pm 5.84$

$84.63 \pm 4.32$

$17.46 \pm 7.65$

$20.03 \pm 8.31$

$10.90 \pm 5.14$

$0.32 \pm 0.26$

$0.66 \pm 0.36$

$1.41 \pm 0.30$

$1.51 \pm 0.48$

$2.05 \pm 0.41$

$83.55 \pm 5.72$

$84.61 \pm 5.84$

$16.33 \pm 8.42$

$17.46 \pm 7.65$

$1.17 \pm 1.53$

$10.90 \pm 5.14$

$1.45 \pm 0.34$

$0.66 \pm 0.36$

$2.11 \pm 0.44$

$T O=5 \mathrm{~s}$ time-out; $H L=$ houselight. Data are expressed as mean $\pm \mathrm{SD}$

ITI: $F[2,22]=10.08, p<0.001)$. Premature responses were significantly and differentially increased in the UA and TR group (Fig. 5c, ITI: $F[2,42]=23.42, p<0.001$; group $\times$ ITI: $F[2,22]=8.3, p<0.001$ ), with the TR group showing the strongest increase in premature responding at the longest ITI.
Variable SDs between 1, 0.5, and $0.2 \mathrm{~s}$ significantly affected accuracy to the same extent in both protocols, with a decrease at shorter SDs (Fig. 5d, SD: $F[2,42]=68.45$, $p<0.001$; group $\times \mathrm{SD}: F[2,22], p=0.3)$. Omissions were differentially increased in the groups, with increments in
Fig. 2 Training time to SD1 criterion performance is less than 1 week in home-cage 5CSRTT protocols. a Number of training sessions (conventional protocol) or training days (home-cage protocol) to SD1 criterion in the task. b Total number of trials to reach SD1 criterion. CT, conventional training; UA, unlimited access; TR, time-restricted. $\mathbf{c}$ Number of trials to reach criterion performance during each learning stage of the task for the

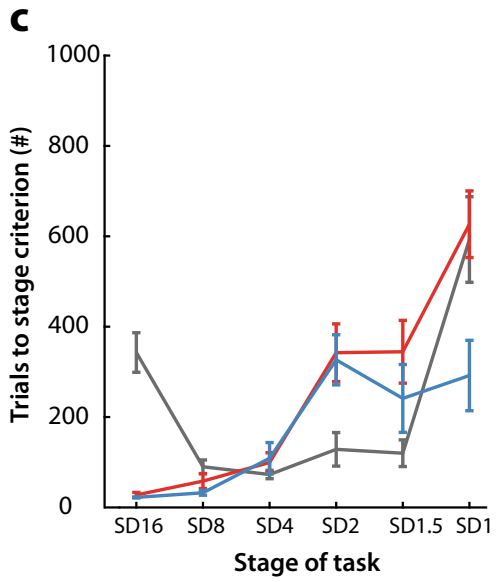

different protocols. Data are expressed as mean \pm SEM. $n=14$ for conventional training (CT), $n=12$ for both the unlimited access (UA), and time-restricted group (TR). ${ }^{*} p<0.01$ Wilcoxon rank-sum test or $t$ test between UA and TR protocol. Conventional training separated by vertical dashed line 
Fig. 3 Behavioral performance over the light/dark cycle in the unlimited access group. a-d Performance in home-cage 5C unlimited protocol distributed over the day. Time is indicated as hour of the day, and time bins in shading represent the dark phase of the day. $* p<0.05$ paired $t$ test light vs dark phase. $n=12$. Data are expressed as mean \pm SEM
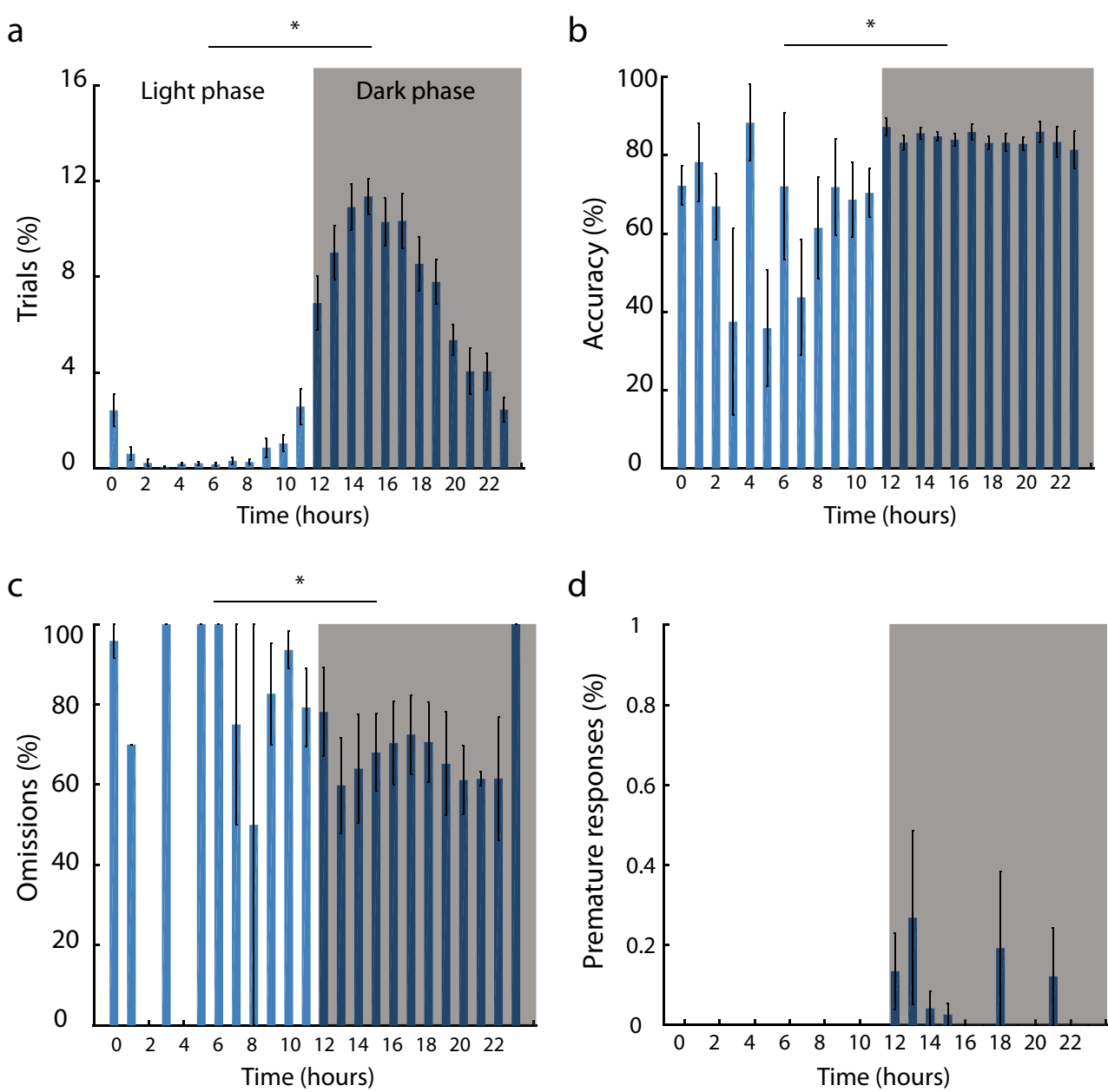

the UA group at 0.5 and $0.2 \mathrm{~s}$, whereas only the shortest $\mathrm{SD}$ increased omissions in the TR group (Fig. 5e, SD: $F$ $[2,42]=81.34, p<0.001 ; \mathrm{SD} \times$ group: $F[2,22]=7.14$, $p<0.001)$. Premature responses were not affected in either group by varying the $\mathrm{SD}$ (Fig. 5f, SD: $F[2,42]=0.57, p=$ 0.57 ; $\mathrm{SD} \times$ group: $F[2,22]=0.42, p=0.66)$. Taken together, these data show that varying ITIs mainly affected premature responding in both groups, with subtle effects on omissions in the UA group, whereas variable SD conditions caused decrements in accuracy and increments in omissions in both UA and TR group, similar to what has been reported previously in the conventional 5-CSRTT (Robbins 2002; Bari et al. 2008).

\section{Effects of scopolamine on behavioral performance}

During the scopolamine experiments, one animal in the TR group and one animal in the UA group did not start trials after the high dose $(0.3 \mathrm{mg} / \mathrm{kg})$ and were therefore excluded from analyses. For further pharmacological validation of the SP-5CSRTT protocols, we used scopolamine, a muscarinic acetylcholine receptor antagonist. Scopolamine has previously been shown to affect multiple aspects of executive functioning in both the conventional 5-CSRTT in rats, as well as the automated home-cage 5-CSRTT in mice (Pattij et al. 2007; Hodges et al. 2009; Remmelink et al. 2017). The 2.5-h session of the TR group was analyzed in five 30-min blocks considering the short half-life of scopolamine in rats (Lyeth et al. 1992). For the UA group, we analyzed the first $2.5 \mathrm{~h}$ in 30 min blocks.

In the TR SP-5-CSRTT protocol, scopolamine decreased the number of started trials throughout the 2.5-h session and to a similar extent across the 30-min blocks. The number of started trials also decreased over time in a session (Fig. 6a, dose: $F[2,9]=18.44, p<0.001$, time: $F[4,9]=5.37, p<0.01$, dose $\times$ time: $F[8,9]=1.06, p=0.4)$. The high dose of scopolamine, $0.3 \mathrm{mg} / \mathrm{kg}$, decreased accuracy of responding in the first and last half hour block of the session (Fig. 6b, dose: $F$ $[2,9]=4.88, p<0.05$, time: $F[4,9]=5.59, p<0.01$, dose $\times$ time: $F[8,9]=2.56, p<0.05)$. Omissions were dosedependently increased by scopolamine throughout the entire session (Fig. 6c, dose: $F[2,9]=13.59, p<0.001$, time: $F$ 
a

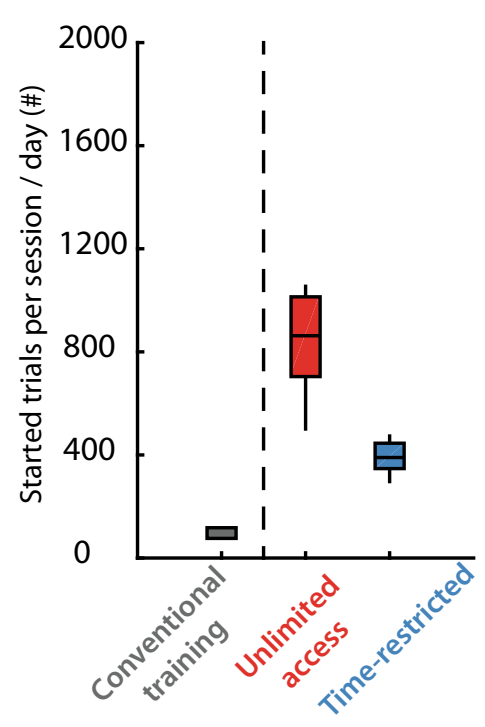

d

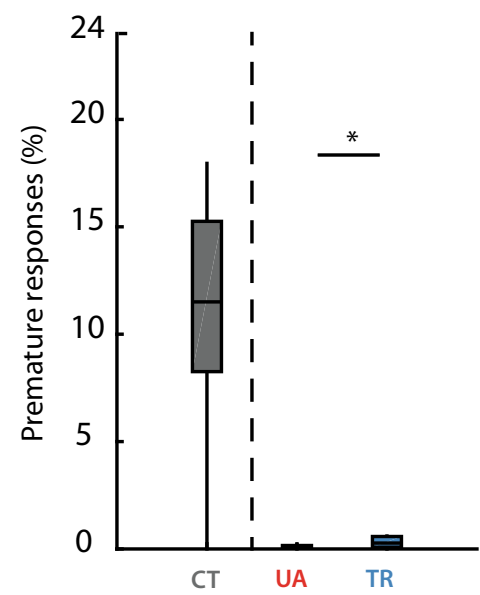

b

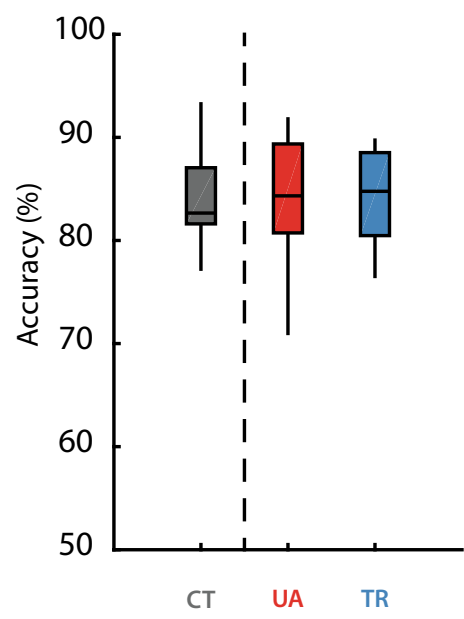

e

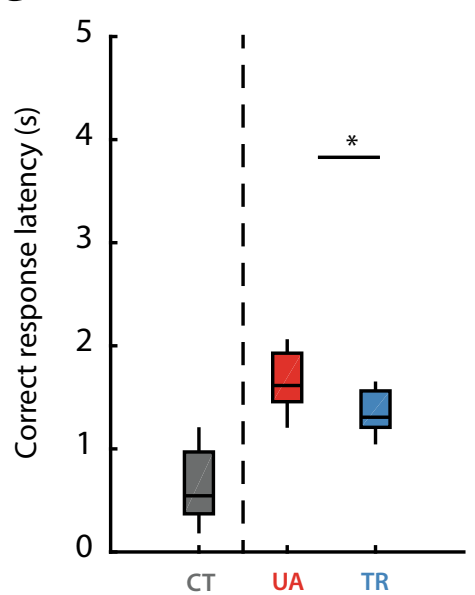

C

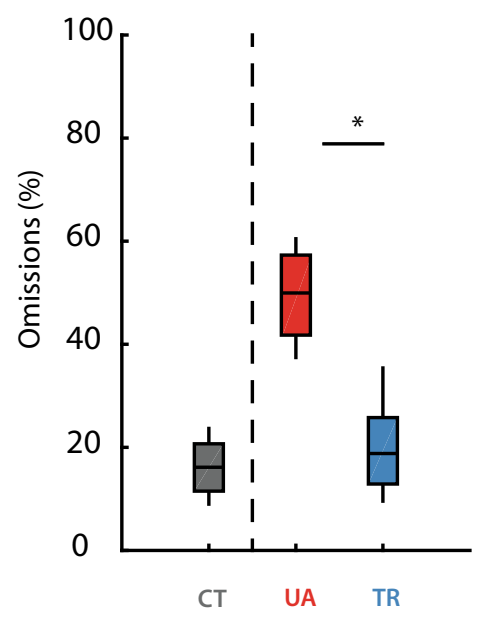

Fig. 4 Behavioral performance at SD1 in the conventional 5CSRTT task and home-cage 5CSRTT protocols. a Number of started trials per session (conventional 5C) or per day (home-cage protocols). b-f Performance at $\mathrm{SD} 1$, data displayed for the measured task parameters. $* p<0.05 t$ test

$[4,9]=4.06, p<0.01$, dose $\times$ time: $F[8,9]=1.65, p=0.13)$. Scopolamine specifically increased premature responses during the first half hour block at the highest dose, and overall premature responding decreased over time (Fig. 6d, dose: $F$ $[2,9]=2.25, p=0.13$, time: $F[4,9]=9.26, p<0.001$, dose $\times$ time: $F[8,9]=2.5, p<0.05$ ). Correct-response latencies were increased by scopolamine throughout the session (Fig. 6e, dose: $F[2,9]=6.72, p<0.01$, time: $F[4,9]=2.87, p<0.05$, dose $\times$ time: $F[8,9]=1.87, p=0.08)$. Magazine latencies were not affected by administration of scopolamine, but increased over the half hour time blocks (Fig. 6f, dose: $F[2,9]=$ 1.71, $p=0.21$, time: $F[4,9]=10.2, p<0.001$, dose $\times$ time: $F$ $[8,9]=1.09, p=0.38)$. In conclusion, scopolamine affected attention and impulse control performance in the TR SP-5CSRTT similarly as has been reported previously for the between UA and TR protocol. Conventional training separated by vertical dashed line. CT, conventional training; UA, unlimited access; TR, timerestricted. $n=14$ for conventional training, $n=12$ for each home-cage $5 \mathrm{C}$ group. Data are expressed as mean \pm SEM

conventional 5-CSRTT and SP-5-CSRTT in mice (Pattij et al. 2007; Hodges et al. 2009; Remmelink et al. 2017).

In the UA SP-5-CSRTT protocol, scopolamine did not affect the number of started trials, but this variable was affected by time (Fig. 7a, dose: $F[2,10]=1.33, p=0.29$, time: $F[4,10]=$ $8.87, p<0.001$, dose $\times$ time: $F[8,10]=0.82, p=0.59$ ). Accurate responding was not affected by scopolamine administration or by time (Fig. $7 b$, dose: $F[2,10]=0.11, p=0.9$, time: $F[4,10]=1.19, p=0.33$, dose $\times$ time: $F[8,10]=1.01, p=$ 0.43). Scopolamine did not alter omissions in the task, which were affected by time (Fig. 7c, dose: $F[2,10]=2.13, p=0.15$, time: $F[4,10]=5.4, p<0.01$, dose $\times$ time: $F[8,10]=1.49, p=$ $0.18)$. Premature responses were not affected by either scopolamine or time (Fig. $7 \mathrm{~d}$, dose: $F[2,10]=3.23, p=0.06$, time: $F$ $[4,10]=0.94, p=0.45$, dose $\times$ time: $F[8,10]=0.77, p=0.63)$. 

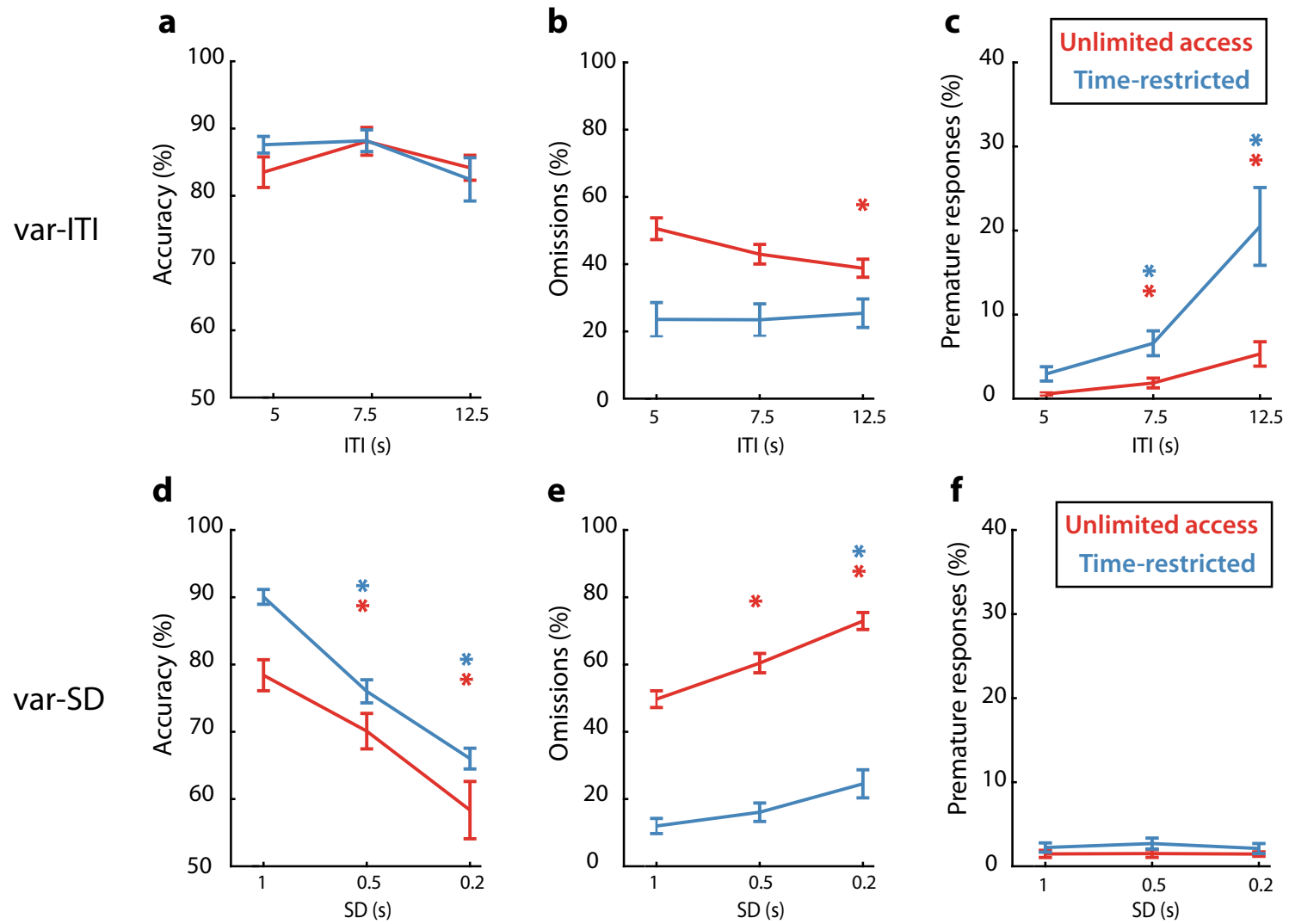

Fig. 5 Behavioral performance in home-cage $5 \mathrm{C}$ is affected by varying SD and ITI. a-c Effect of varying the inter-trial interval (var-ITI) on selected task parameters. $* p<0.05$ FDR-corrected paired $t$ test vs ITI $=5 \mathrm{~s}$. The color of the asterisk depicts in which group the difference is detected. $\mathbf{d}-\mathbf{f}$ Effect of varying the stimulus duration (var-SD) on

selected task parameters. $* p<0.05$ FDR-corrected paired $t$ test vs $\mathrm{SD}=1 \mathrm{~s}$. The color of the asterisk depicts which group the difference is detected. UA, unlimited access $(n=12)$; TR, time-restricted $\operatorname{protocol}(n=$ 11). Data are expressed as mean \pm SEM

Scopolamine increased correct-response latencies throughout the session (Fig. 7e, dose: $F[2,10]=4.31, p<0.05$, time: $F$ $[4,10]=0.81$, dose $\times$ time: $F[8,10]=1.17, p=0.33)$. Finally, magazine latencies were not altered by scopolamine administration (Fig. 7f, dose: $F[2,10]=1.51, p=0.25$, time: $F[4,10]=$ $1.95, p=0.13$, dose $\times$ time: $F[8,10]=1, p=0.44$ ). In summary, scopolamine failed to affect attention and inhibitory control in the UA SP-5-CSRTT protocol but increased correct-response latencies.

\section{Discussion}

We present an automated home-cage-based version of the 5CSRTT for rats, as was previously developed for mice (Remmelink et al. 2017). Our main findings are that in the SP-5-CSRTT, training time was less than 1 week and that animals gained weight during training without the necessity of prior food restriction. SP-5-CSRTT was sensitive to behavioral challenges in similar fashion as demonstrated in the conventional 5-CSRTT, whereas only in

the TR-group, pharmacological interventions with scopolamine were effective.

Conventional 5-CSRTT requires long training periods (Granon et al. 2000; Hahn and Shoaib 2002; Bari et al. 2008; Luchicchi et al. 2016). Rats with UA to SP-5-CSRTT finished training in less than 4 days, while rats with TR access finished within 1 week. The training time reduction most likely results from the increased number of trials that rats performed each day. Interestingly, the total number of trials to reach SD1 criterion was reduced for the TR protocol. A closer look at the number of required trials per stage revealed that learning dynamics differed between protocols. Rats trained in the TR protocol required less trials to learn the final stage under SD1 conditions. One factor contributing to this different rate of learning could be the continuous food availability in the UA protocol. This might increase satiety and decrease motivation, possibly reflected by the increase in percentage omissions as discussed below.

Baseline performance in SP-5-CSRTT differed on several parameters between protocols. Rats in the UA group started trials preferably in the dark phase compared with the light phase (Loos et al. 2014; Remmelink et al. 2017; Rivalan et al. 2017). 


\section{Saline $\quad 0.1 \mathrm{mg} / \mathrm{kg} \square 0.3 \mathrm{mg} / \mathrm{kg}$}

a

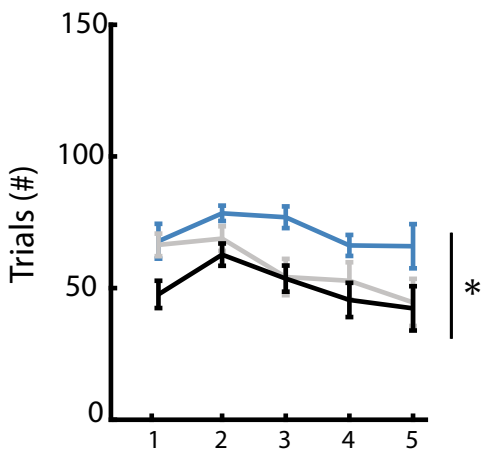

d

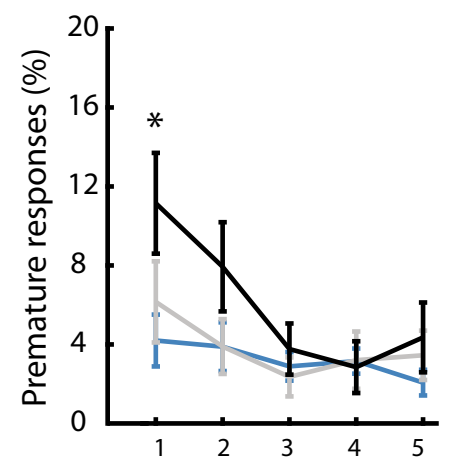

b

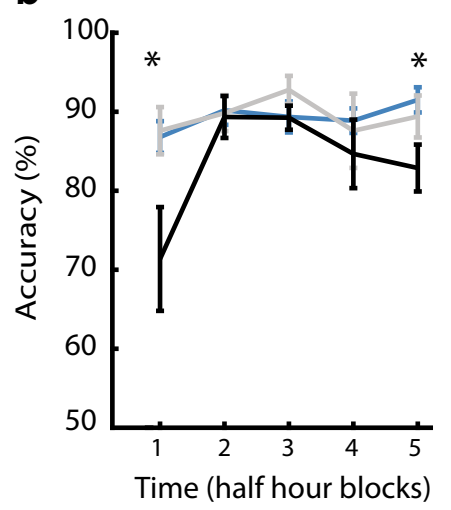

e

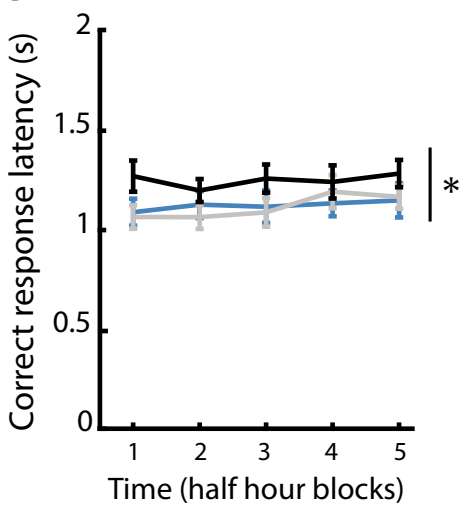

C

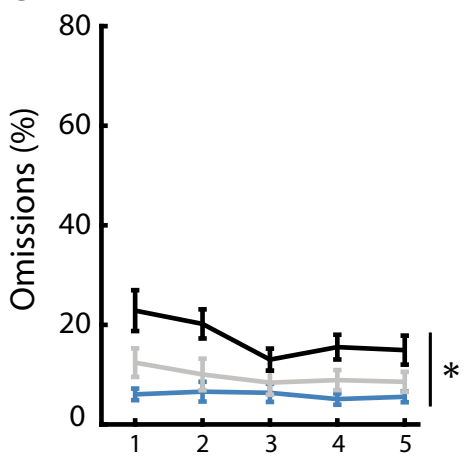

f

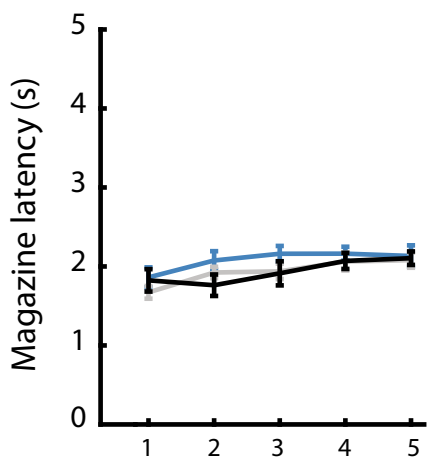

Fig. 6 Scopolamine affects cognitive parameters in time-restricted (TR) home-cage $5 \mathrm{C}$ task. a Scopolamine decreased the number of started trials over the session. $* p<0.05$ main effect dose repeated-measures ANOVA. b Accuracy was reduced by the highest dose of scopolamine in the first and fifth time block. $* p<0.05$ FDR-corrected Wilcoxon signed-rank test $0.3 \mathrm{mg} / \mathrm{kg}$ vs saline. $\mathbf{c}$ Scopolamine increased the percentage of omitted trials over the session. $* p<0.05$. d Scopolamine increased the percentage

Omissions were dramatically increased in the UA group, possibly resulting from reduced motivation or reduced salience of visual cues in light surroundings. Restriction of trial accessibility (TR) strongly reduced levels of omissions in the SP-5-CSRTT. In human subjects, similar observations have been made regarding time limits in motivation and task performance. When subjects were given twice the necessary amount of time needed for solving an addition task, it not only took longer to complete the task, but easier task goals were set (Locke 1968). To our knowledge, our study is the first to directly compare effects of time limits on task performance in rodents. In addition, levels of premature responding were lower in both home-cage 5-CSRTT protocols. In mice, no differences in levels of premature responding or increases in omissions were reported between conventional training and home-cage 5-CSRTT (Remmelink et al. 2017). This might be due to subtle differences in task design, such as a longer eat-ITI in the mouse SP-5-CSRTT protocol or to inherent of premature responses in the first time block of the session. $* p<0.05$ FDR-corrected Wilcoxon signed-rank test $0.3 \mathrm{mg} / \mathrm{kg}$ vs saline. e Correctresponse latency was increased after scopolamine administration. * $p<0.05$ main effect dose repeated-measures ANOVA. f Scopolamine did not affect the magazine latency. $n=10$. Data are expressed as mean \pm SEM

differences in premature responding strategies between mice and rats as previously reported (Young et al. 2013; Cope et al. 2016).

A potential caveat could be differences in signaling of response errors in the tasks. In the conventional 5-CSRTT, error are punished by time-out periods signaled through house-light extinction (Bari et al. 2008). In SP-5-CSRTT, time-out periods were signaled by turning on the house-light. Despite this, behavioral challenges, such as varying ITI or SD, resulted in similar effects to conventional 5-CSRTT (Bari et al. 2008; Saund et al. 2017; Schippers et al. 2017). Varying the ITI led to increased premature responding and decreased omissions. Increasing ITI durations has previously been reported to lower omissions in rats (Schippers et al. 2017), yet increases in omissions have also been shown (Chudasama et al. 2003; Saund et al. 2017). This may result from shorter limited hold periods urging faster responses following stimulus presentation. Shortening SDs decreased ac- 
Fig. 7 Scopolamine does not affect attention and inhibitory control in unlimited access (UA) home-cage 5C task. a-f Effect of scopolamine on selected task parameters. Scopolamine increased the correct-response latency but did not alter other parameters. * $p<0.05$ main effect dose repeated-measures ANOVA. $n=$ 11. Data are expressed as mean \pm SEM

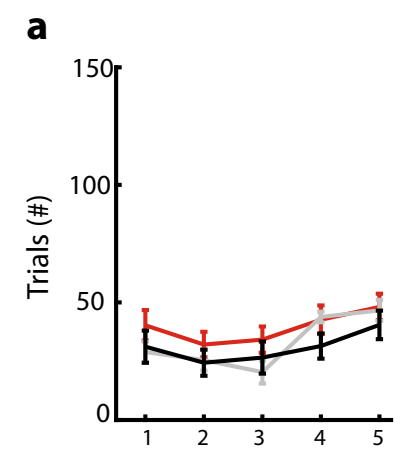

d

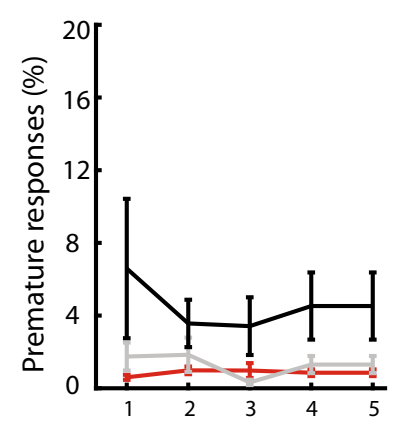

Saline

$0.1 \mathrm{mg} / \mathrm{kg}$

$0.3 \mathrm{mg} / \mathrm{kg}$

b

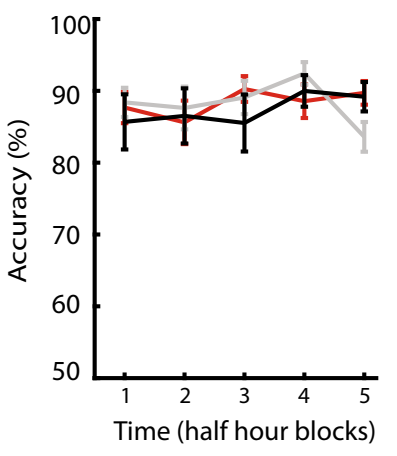

C
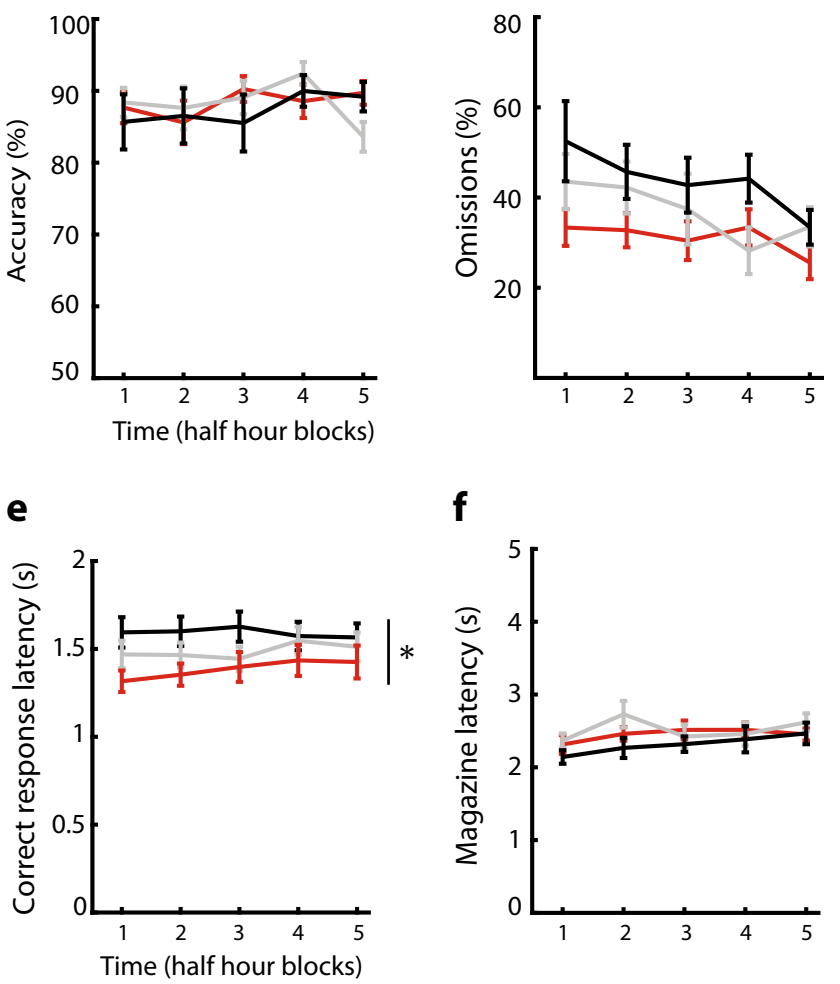

f

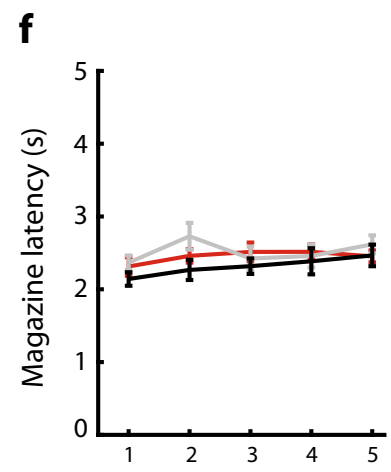

curate choice as well as increased the percentage of omitted trials (Bari et al. 2008; Counotte et al. 2011). Thus in SP-5-CSRTT, variable ITIs mainly affected impulsive responding, while variable SDs mainly affected attentional performance. The validity of the SP-5-CSRTT for drug screening was demonstrated by scopolamine (muscarinic acetylcholine receptor antagonist) challenges, which have been well characterized in both mice and rats in conventional 5-CSRTT (Pattij et al. 2007; Hodges et al. 2009; Remmelink et al. 2017). Reported effects of scopolamine on attention and inhibitory control were replicated in the TR protocol, but not in the UA protocol. Scopolamine decreased accuracy mainly in the first half hour block of the TR protocol. Additionally, premature responding was specifically increased in the first half hour, in line with plasma half-life of scopolamine (Lyeth et al. 1992). In contrast, the number of started trials, correct-response latency, and omissions were affected throughout the entire session. Whereas in mice, scopolamine has been shown to robustly decrease accurate responding, in rats, results are inconsistent in literature (Pattij et al. 2007; Remmelink et al. 2017). Similar to our findings, decrements in accuracy have been reported (Jones and Higgins 1995; Mirza and Stolerman 2000). In contrast, several other studies found no effect of scopolamine on accurate choice in rats (Jäkälä et al. 1992; Higgs et al. 2000; Hodges et al. 2009). Interestingly, effects of scopolamine on accurate choice were mainly found under more challenging conditions, such as white noise distraction (Jones and Higgins 1995), or by reducing SDs (Mirza and Stolerman 2000). In the present study, animals were tested with long, variable ITI sessions to increase task unpredictability. Possibly, scopolamine affects attentional parameters when the cognitive load is increased. Alternatively, scopolamine effects may be rat strain-dependent, similar to effects of nicotine in the 5-CSRTT (Mirza and Bright 2001).

In the TR protocol, scopolamine also increased omission rate and decreased number of started trials throughout the entire 2.5-h duration of the session, suggesting decreased motivation. This could indicate that scopolamine impacts cognitive functions for a short period after injection, whereas its effects on motivation are longer-lasting. Reduced motivation may also be the main effect of scopolamine in the UA protocol, where we only found an increase in correct-response latency and no effect on accurate choice and premature responding. This finding is in contrast with the results in the TR protocol in rats and findings in mice where scopolamine did affect impulsivity and attentional processes in the SP-5CSRTT (Remmelink et al. 2017). We hypothesize that the lower number of started trials in a specific time bin and the higher level of omissions in the UA protocol reflect diminished engagement in the task as mentioned above. This would make the UA protocol less valid for pharmacological testing than conventional 5-CSRTT or the TR protocol, especially for drugs with a short half-life like scopolamine. Careful 
consideration for the selection of training and testing protocol is thus necessary based on the research question.

One remaining question is how the SP-5-CSRTT contributes to habitual versus goal-directed responding in this task compared with conventional training. Learning of this task is based on reinforcement and continuation of similar task contingencies after reaching criterion performance results in stimulus-response habits or overtraining (Jog et al. 1999). This habitual form of responding lacks signs of cognitive contributions and exhibits insensitivity to value of the outcome and to changes in actionoutcome contingencies (Yin and Knowlton 2006; Smith and Graybiel 2016). To our knowledge, the transition from goaldirected behavior to habitual responding has not directly been studied in the 5-CSRTT, by, for instance, changing actionoutcome contingencies, i.e., by rewarding only $50 \%$ of correct responses and assess effects on performance. Since the SP-5CRTT protocols allow the animals to perform more trials per day, they will potentially overtrain more quickly in the task. We therefore recommend that testing of pharmacological compounds takes place in cognitively challenging sessions, which require the animal to break fixed response routines.

A potential caveat of the SP-5-CSRTT is that rats were housed individually in CombiCages. Social isolation in rats can lead to increased stress levels and altered neuroendocrine state, particularly during early weaning (Serra et al. 2000; Weiss et al. 2004; Weintraub et al. 2010), which has been found to impact executive functions in rats (Kirkpatrick et al. 2013; Wang et al. 2017). Notably, these effects are most pronounced when social isolation occurs following early weaning, for instance starting at postnatal day 21. Our SP5CSRTT training started when animals were at least 63 days old. It has recently been shown that prolonged individual housing of adult rats did not influence corticosterone concentration, hippocampal long-term potentiation measurements, and object place recognition (Riga et al. 2017). Combined with the restricted amount of experimental time, self-paced training, and less food restriction, stress effects are most likely limited in SP-5CSRTT. Social housing and home-cage testing can be combined in rats (Rivalan et al. 2017) and are important points of improvement of the SP-5-CSRTT. Secondly, the accelerated learning rate and format of the task might influence the neurobiological correlates of behavioral performance when compared with the conventional 5-CSRTT. Nevertheless, home-cage-based training of rats in the SP-5CSRTT provides a rapid and reliable alternative for conventional training in the task to measure attention and motor impulsivity. The short training time opens up new possibilities and allows, for instance, specific testing of young or adolescent rats, which in the conventional paradigm is not possible due to time constraints. Thereby, SP-5CSRTT is highly suited to address questions involving pharmacological challenges or to investigate the physiological mechanisms of attention and motor impulsivity during limited time windows.
Acknowledgements We would like to thank the VU University Amsterdam Fine Mechanics Workshop for excellent technical support.

Funding information H.D.M. received funding for this work from the Netherlands Organization for Scientific Research (NWO VICI grant 865.13.002) and EU H2020 (Grant Agreement H2020 HBP 720270).

Open Access This article is distributed under the terms of the Creative Commons Attribution 4.0 International License (http:// creativecommons.org/licenses/by/4.0/), which permits unrestricted use, distribution, and reproduction in any medium, provided you give appropriate credit to the original author(s) and the source, provide a link to the Creative Commons license, and indicate if changes were made.

Publisher's note Springer Nature remains neutral with regard to jurisdictional claims in published maps and institutional affiliations.

\section{References}

Bari A, Dalley JW, Robbins TW (2008) The application of the 5-choice serial reaction time task for the assessment of visual attentional processes and impulse control in rats. Nat Protoc 3:759-767. https://doi.org/10.1038/nprot.2008.41

Benjamini Y, Hochberg Y (1995) Controlling the false discovery rate: a practical and powerful approach to multiple testing. J R Stat Soc Ser B Methodol 57(1):289-300

Bhandari J, Daya R, Mishra RK (2016) Improvements and important considerations for the 5-choice serial reaction time task-an effective measurement of visual attention in rats. J Neurosci Methods 270:17-29. https://doi.org/10.1016/j.jneumeth.2016.06.002

Blondeau C, Dellu-Hagedorn F (2007) Dimensional analysis of ADHD subtypes in rats. Biol Psychiatry 61:1340-1350. https://doi.org/10. 1016/j.biopsych.2006.06.030

Bohacek J, Daniel JM (2007) Increased daily handling of ovariectomized rats enhances performance on a radial-maze task and obscures effects of estradiol replacement. Horm Behav 52:237-243. https://doi. org/10.1016/j.yhbeh.2007.04.010

Castellanos FX, Tannock R (2002) Neuroscience of attention-deficit/hyperactivity disorder: the search for endophenotypes. Nat Rev Neurosci 3:617-628. https://doi.org/10.1038/nrn896

Chudasama Y, Passetti F, Rhodes SE et al (2003) Dissociable aspects of performance on the 5-choice serial reaction time task following lesions of the dorsal anterior cingulate, infralimbic and orbitofrontal cortex in the rat: differential effects on selectivity, impulsivity and compulsivity. Behav Brain Res 146:105-119. https://doi.org/10. 1016/j.bbr.2003.09.020

Cope ZA, Halberstadt AL, van Enkhuizen J, Flynn AD, Breier M, Swerdlow NR, Geyer MA, Young JW (2016) Premature responses in the five-choice serial reaction time task reflect rodents??? Temporal strategies: evidence from no-light and pharmacological challenges. Psychopharmacology 233:3513-3525. https://doi.org/ 10.1007/s00213-016-4389-4

Counotte DS, N a G, Li KW et al (2011) Lasting synaptic changes underlie attention deficits caused by nicotine exposure during adolescence. Nat Neurosci 14:417-419. https://doi.org/10.1038/nn.2770

Deutsch-Feldman M, Picetti R, Seip-Cammack K et al (2015) Effects of handling and vehicle injections on adrenocorticotropic and corticosterone concentrations in Sprague-Dawley compared with Lewis rats. J Am Assoc Lab Anim Sci 54:35-39. https://doi.org/10.1371/ journal.pone. 0124488

Granon S, Passetti F, Thomas KL et al (2000) Enhanced and impaired attentional performance after infusion of D1 dopaminergic receptor 
agents into rat prefrontal cortex. J Neurosci 20:1208-1215. https:// doi.org/10.1038/nrg1871

Hahn B, Shoaib M, Stolerman IP (2002) Nicotine-induced enhancement of attention in the five-choice serial reaction time task: the influence of task demands. Psychopharmacology 162:129-137. https://doi. org/10.1007/s00213-002-1005-6

Higgs S, Deacon RMJ, Rawlins JNP (2000) Effects of scopolamine on a novel choice serial reaction time task. Eur J Neurosci 12:17811788. https://doi.org/10.1046/j.1460-9568.2000.00047.x

Hodges DB, Lindner MD, Hogan JB et al (2009) Scopolamine induced deficits in a battery of rat cognitive tests: comparisons of sensitivity and specificity. Behav Pharmacol 20:237-251. https://doi.org/10. 1097/FBP.0b013e32832c70f5

Jäkälä P, Sirviö J, Jolkkonen J, Riekkinen P Jr, Acsady L, Riekkinen P (1992) The effects of p-chlorophenylalanine-induced serotinin synthesis inhibition and muscarinic blockade on the performance of rats in a 5-choice serial reaction time task. Behav Brain Res 51:29-40. https://doi.org/10.1016/S0166-4328(05)80309-2

Jog MS, Kubota Y, Connolly CI, Hillegaart V, Graybiel AM (1999) Building neural representations of habits. Science (80-) 286:17451749. https://doi.org/10.1126/science.286.5445.1745

Jones DNC, Higgins GA (1995) Effect of scopolamine on visual attention in rats. Psychopharmacology 120:142-149. https://doi.org/10.1007/ BF02246186

Kirkpatrick K, Marshall AT, Clarke J, Cain ME (2013) Environmental rearing effects on impulsivity and reward sensitivity. Behav Neurosci 127:712-724. https://doi.org/10.1037/a0034124

Locke EA (1968) Toward a theory of task motivation and incentives. Organ Behav Hum Perform 3:157-189. https://doi.org/10.1016/ 0030-5073(68)90004-4

Loos M, Koopmans B, Aarts E, Maroteaux G, van der Sluis S, NeuroBSIK Mouse Phenomics Consortium, Verhage M, Smit AB (2014) Sheltering behavior and locomotor activity in 11 genetically diverse common inbred mouse strains using home-cage monitoring. PLoS One 9:1-9. https://doi.org/10.1371/journal.pone.0108563

Luchicchi A, Mnie-Filali O, Terra H, Bruinsma B, de Kloet SF, Obermayer J, Heistek TS, de Haan R, de Kock CPJ, Deisseroth K, Pattij T, Mansvelder HD (2016) Sustained attentional states require distinct temporal involvement of the dorsal and ventral medial prefrontal cortex. Front Neural Circuits 10:1-14. https://doi.org/10. 3389/fncir.2016.00070

Luck SJ, Gold JM (2008) The construct of attention in schizophrenia. Biol Psychiatry 64:34-39. https://doi.org/10.1016/j.biopsych.2008. 02.014

Lyeth BG, Ray M, Hamm RJ, Schnabel J, Saady JJ, Poklis A, Jenkins LW, Gudeman SK, Hayes RL (1992) Postinjury scopolamine administration in experimental traumatic brain injury. Brain Res 569: 281-286. https://doi.org/10.1016/0006-8993(92)90640-U

Mirza NR, Bright JL (2001) Nicotine-induced enhancements in the fivechoice serial reaction time task in rats are strain-dependent. Psychopharmacology 154:8-12. https://doi.org/10.1007/ s002130000605

Mirza NR, Stolerman IP (2000) The role of nicotinic and muscarinic acetylcholine receptors in attention. Psychopharmacology 148: 243-250. https://doi.org/10.1007/s002130050048

Pattij T, Janssen MCW, Loos M, Smit AB, Schoffelmeer ANM, van Gaalen MM (2007) Strain specificity and cholinergic modulation of visuospatial attention in three inbred mouse strains. Genes Brain Behav 6:579587. https://doi.org/10.1111/j.1601-183X.2006.00284.X

Remmelink E, Chau U, Smit AB, Verhage M, Loos M (2017) A oneweek 5-choice serial reaction time task to measure impulsivity and attention in adult and adolescent mice. Sci Rep 7:42519. https://doi. org/10.1038/srep42519
Riga D, Kramvis I, Koskinen MK, van Bokhoven P, van der Harst JE, Heistek TS, Jaap Timmerman A, van Nierop P, van der Schors RC, Pieneman AW, de Weger A, van Mourik Y, Schoffelmeer ANM, Mansvelder HD, Meredith RM, Hoogendijk WJG, Smit AB, Spijker S (2017) Hippocampal extracellular matrix alterations contribute to cognitive impairment associated with a chronic depressive-like state in rats. Sci Transl Med 9:eaai8753. https://doi.org/10.1126/scitranslmed.aai8753

Rivalan M, Munawar H, Fuchs A, Winter Y (2017) An automated, experimenter-free method for the standardised, operant cognitive testing of rats. PLoS One 12:1-19. https://doi.org/10.1371/journal. pone. 0169476

Robbins TW (2002) The 5-choice serial reaction time task: behavioural pharmacology and functional neurochemistry. Psychopharmacology 163:362-380. https://doi.org/10.1007/s00213-002-1154-7

Sänger J, Bechtold L, Schoofs D et al (2014) The influence of acute stress on attention mechanisms and its electrophysiological correlates. Front Behav Neurosci 8:353. https://doi.org/10.3389/fnbeh.2014.00353

Saund J, Dautan D, Rostron C, Urcelay GP, Gerdjikov TV (2017) Thalamic inputs to dorsomedial striatum are involved in inhibitory control: evidence from the five-choice serial reaction time task in rats. Psychopharmacology (Berl) 1-9 234:2399-2407. https://doi. org/10.1007/s00213-017-4627-4

Schippers MC, Bruinsma B, Gaastra M, Mesman TI, Denys D, de Vries TJ, Pattij T (2017) Deep brain stimulation of the nucleus accumbens core affects trait impulsivity in a baseline-dependent manner. Front Behav Neurosci 11:52. https://doi.org/10.3389/fnbeh.2017.00052

Serra M, Pisu MG, Littera M, Papi G, Sanna E, Tuveri F, Usala L, Purdy RH, Biggio G (2000) Social isolation-induced decreases in both the abundance of neuroactive steroids and $\mathrm{GABA}(\mathrm{A})$ receptor function in rat brain. J Neurochem 75:732-740. https://doi.org/10.1046/j. 1471-4159.2000.0750732.x

Smith KS, Graybiel AM (2016) Habit formation. Dialogues Clin Neurosci 18:33-43. https://doi.org/10.1111/clr.12458_111

Sorge RE, Martin LJ, Isbester KA, Sotocinal SG, Rosen S, Tuttle AH, Wieskopf JS, Acland EL, Dokova A, Kadoura B, Leger P, Mapplebeck JCS, McPhail M, Delaney A, Wigerblad G, Schumann AP, Quinn T, Frasnelli J, Svensson CI, Sternberg WF, Mogil JS (2014) Olfactory exposure to males, including men, causes stress and related analgesia in rodents. Nat Methods 11:629-632. https://doi.org/ 10.1038/nmeth.2935

Wang MZ, Marshall AT, Kirkpatrick K (2017) Differential effects of social and novelty enrichment on individual differences in impulsivity and behavioral flexibility. Behav Brain Res 327:54-64. https:// doi.org/10.1016/j.bbr.2017.03.028

Weintraub A, Singaravelu J, Bhatnagar S (2010) Enduring and sex-specific effects of adolescent social isolation in rats on adult stress reactivity. Brain Res 1343:83-92. https://doi.org/10.1016/j.brainres.2010.04.068

Weiss IC, Pryce CR, Jongen-Rêlo AL, Nanz-Bahr NI, Feldon J (2004) Effect of social isolation on stress-related behavioural and neuroendocrine state in the rat. Behav Brain Res 152:279-295. https://doi. org/10.1016/j.bbr.2003.10.015

Yin HH, Knowlton BJ (2006) The role of the basal ganglia in habit formation. Nat Rev Neurosci 7:464-476. https://doi.org/10.1038/ nrn1919

Young JW, Jentsch JD, Bussey TJ, Wallace TL, Hutcheson DM (2013) Consideration of species differences in developing novel molecules as cognition enhancers. Neurosci Biobehav Rev 37:2181-2193. https://doi.org/10.1016/j.neubiorev.2012.10.002 